\title{
COMMENT
}

\section{A glass half-full: defining ventilator-associated pneumonia in the neonatal intensive care unit}

\author{
Emily D. Whitesel ${ }^{1}$ and Munish Gupta ${ }^{1}$ \\ Pediatric Research (2020) 87:1155-1156; https://doi.org/10.1038/s41390-020-0884-8
}

Avoidance of hospital-acquired infections (HAls) may be one of the most dramatic successes of recent quality improvement efforts in health care. In neonatal intensive care units (NICUs), as in medicine in general, this success story has shifted an old view of HAls being unavoidable to a view that HAls are preventable. This may be best exemplified by the experience with central line-associated bloodstream infections (CLABSIs), with significant reductions in NICU CLABSI rates reported locally and nationally and numerous NICUs reporting sustained CLABSI rates of zero. ${ }^{1,2}$

Unfortunately, the experience with ventilator-associated pneumonia (VAP) in the NICUs has not followed a similar path, and widespread reductions in neonatal VAP rates have not been documented. ${ }^{3}$ What explains the different experiences with CLABSI and VAP, particularly in neonatal intensive care? One major contributing factor is eloquently described by Dr. Ergenekon and Dr. Cataltepe in this journal: the lack of a clear definition and diagnostic test for VAP in newborns. ${ }^{4}$ Unlike VAP, CLABSI has had relatively clear definitions and diagnostic criteria for many years. The most commonly used CLABSI definitions are from the National Healthcare Safety Network (NHSN) of the Centers for Disease Control and Prevention (CDC); while not perfect, these definitions have contributed to the reduction in CLABSI by allowing effective benchmarking, supporting the identification of risk factors, and enabling assessment of the impact of improvement efforts. ${ }^{5}$

The history of definitions for VAP is different. The CDC has included nosocomial pneumonia and VAP in its HAI surveillance platforms since the 1970s-1980s. However, concerns quickly arose about the reliability and objectivity of the pneumonia and VAP definitions, and despite several definition revisions, these concerns persisted. ${ }^{6}$ This was particularly true for neonatal VAP, where a definition specific to neonates that recognized their unique characteristics and the complexities of prematurity was not provided; rather, diagnostic criteria for infants under 1 year of age were also applied to neonates. Ongoing questions about the validity and utility of the VAP definitions recently led to broad changes in CDC's approach to measures related to mechanical ventilation. In 2013, NHSN replaced surveillance for VAP in adult inpatient locations with surveillance for ventilator-associated events (VAEs); VAE was defined primarily by an increase in support (fraction of inspired oxygen or positive end expiratory pressure) in a mechanically ventilated patient who had been previously stable or improving, and was purposefully meant to be a more general and more objective measure of ventilatorassociated complications than VAP.? By 2014, NHSN also stopped
VAP surveillance in neonatal locations, leaving VAP surveillance only in pediatric areas. While the pediatric definitions of pneumonia and VAP could still be used to identify the source of a bloodstream infection in an NICU patient, neonatal VAP rates would no longer be an NHSN measure. In 2019, NHSN implemented surveillance for pediatric VAEs, with a definition based on increase in oxygen need or mean airway pressure that had been validated in both pediatric and neonatal patients. ${ }^{8}$ Like its adult counterpart, the pediatric VAE measure was designed to capture infectious and non-infectious complications with more precision and objectivity than the previous VAP measure.

The NHSN shift to VAE surveillance suggests a commonly accepted VAP definition may be more elusive than ever. While the new pediatric VAE measure may eventually prove to be effective and valuable, it is not specific for infectious ventilator events. Hence, the message from Dr. Ergenekon and Dr. Cataltepe for focused research in the area of neonatal VAP diagnosis becomes even more imperative. But what can be done in the meantime? While we agree with their editorial that better diagnostic tools are urgently needed, we would also suggest that much can still be done to limit the risk and burden of VAP in neonatal patients based on what we know already.

Dr. Ergenekon and Dr. Cataltepe broadly suggest two important consequences of the lack of a clear neonatal VAP definition: (1) impaired clinical decision making around VAP, with variations in practice and likely unnecessary antibiotic use; and (2) misguided use of VAP as a quality measure. It may be helpful to consider these uses of a definition separately, as they likely have different implications for what an ideal definition would be. A definition of VAP as a quality measure would require clear criteria that can be unambiguously applied to many patients; a diagnosis of VAP in a single patient to determine treatment would likely be based on many factors and clinical judgment. For example, a definition of VAP as a quality measure may require a certain number of neutrophils on a tracheal aspirate fluid sample; in an individual patient, a lesser number of neutrophils combined with other clinical concerns may still be enough to warrant diagnosis and treatment. It is likely that treatment decisions will almost always require assessment of factors not captured by quality measures used for surveillance. This is evident with CLABSI, where NICU CLABSI rates measured by the NHSN criteria do not match well with infection rates assessed by the clinical criteria. ${ }^{9,10}$

With regards to developing a definition for VAP as a quality measure, it is important to recognize differences between measures for quality assessment and measures for quality improvement. ${ }^{11}$ Measures for quality assessment are used to

${ }^{1}$ Department of Neonatology, Beth Israel Deaconess Medical Center, Harvard Medical School, Boston, MA, USA

Correspondence: Munish Gupta (mgupta@bidmc.harvard.edu)

Received: 25 February 2020 Accepted: 4 March 2020

Published online: 2 April 2020 
Table 1. Potential neonatal ventilator-associated pneumonia prevention strategies ${ }^{\mathrm{a}}$.

Meticulous hand hygiene

Elevate head of bed by $15^{\circ}-30^{\circ}$ if tolerated

Use new, sterile ETT for each intubation attempt

Use sterilized laryngoscope

Have at least two NICU staff members present for ETT retaping or repositioning

Avoid unplanned extubation

Use closed in-line suctioning for endotracheal tube suctioning

Minimize duration of invasive mechanical ventilation

Manage patients without sedation when possible

Change ventilator circuit only if visibly soiled or malfunctioning

Minimize breaks in the ventilator circuit

Provide regular oral care with sterile water or mother's milk

Prevent gastric distension

${ }^{a}$ Adapted from Klompas et al. ${ }^{6}$ and Hooven and Polin. ${ }^{20}$

compare performance among hospitals and for benchmarking, and need reliability, reproducibility, and external validity. Measures for quality improvement are used to monitor performance and guide improvement within one center, and need internal consistency and can be targeted to meet the needs of that center.

We agree with Dr. Ergenekon and Dr. Cataltepe that current definitions of neonatal VAP do not have the validity or reliability needed to be used for external quality assessment and benchmarking. This conclusion appears to be widely shared; as outlined above, NHSN no longer tracks VAP as a quality measure in adults or neonates, and VAP is also not endorsed by other major neonatal quality organizations, including Vermont-Oxford Network, the Children's Hospital Neonatal Consortium, and state perinatal quality collaboratives.

On the other hand, the use of VAP as an internal quality improvement measure has been effective. Several reports show NICUs have successfully improved practices and reduced VAP rates using internally consistent measure definitions. ${ }^{12-19}$ These reports and expert consensus have led to generally accepted bundles of practices that can be used to minimize VAP risk and reduce VAP incidence (Table 1). ${ }^{6,20}$ Some of these interventions are extrapolated from literature in older patients, but their basis in physiology and mechanics and their successful use in published quality improvement reports suggest that they are a reasonable target for neonatal practice. Given the significant morbidity and mortality associated with VAP, applying these bundles has the potential to minimize risk and improve outcomes in the neonatal population, advancing clinical care while we await a more standardized definition.

Our lessons learned about VAP in the NICUs, then, may not be that different than our lessons learned about CLABSIs. While we still struggle to develop a reliable and valid VAP definition that can be used at the bedside to guide treatment choices or at the national level to benchmark hospitals, ample experience suggests that we can define VAP well enough to try to prevent it. We support the call for targeted research into neonatal VAP diagnosis and definition. We also encourage neonatal providers to not wait for a better definition to strive towards reducing or eliminating VAP in their own NICUs.

\section{AUTHOR CONTRIBUTIONS}

E.D.W. and M.G. had substantial contributions to conception, design, drafting, and revision of the article, and approved the final version.

\section{ADDITIONAL INFORMATION}

Competing interests: The authors declare no competing interests.

Publisher's note Springer Nature remains neutral with regard to jurisdictional claims in published maps and institutional affiliations.

\section{REFERENCES}

1. Payne, V., Hall, M., Prieto, J. \& Johnson, M. Care bundles to reduce central lineassociated bloodstream infections in the neonatal unit: a systematic review and meta-analysis. Arch. Dis. Child Fetal Neonatal Ed. 103, F422-F429 (2018).

2. Suresh, G. K. \& Edwards, W. H. Central line-associated bloodstream infections in neonatal intensive care: changing the mental model from inevitability to preventability. Am. J. Perinatol. 29, 57-64 (2012).

3. Cernada, M., Brugada, M., Golombek, S. \& Vento, M. Ventilator-associated pneumonia in neonatal patients: an update. Neonatology 105, 98-107 (2014).

4. Ergenekon, E. \& Çataltepe, S. Ventilator-associated pneumonia in the NICU: time to boost diagnostics? Pediatr. Res. https://doi.org/10.1038/s41390-019-0672-5 (2019).

5. Mobley, R. E. \& Bizzarro, M. J. Central line-associated bloodstream infections in the NICU: successes and controversies in the quest for zero. Semin. Perinatol. 41, 166-174 (2017).

6. Klompas, M. et al. Society for Healthcare Epidemiology of America. Strategies to prevent ventilator-associated pneumonia in acute care hospitals: 2014 update. Infect. Control Hosp. Epidemiol. 35, 915-936 (2014).

7. Magill, S. S. et al. Developing a new, national approach to surveillance for ventilator-associated events: executive summary. Infect. Control Hosp. Epidemiol. 34, 1239-1243 (2013).

8. Cocoros, N. M. et al. Pediatric Ventilator-Associated Conditions Study Team. Ventilator-associated events in neonates and children-a new paradigm. Crit. Care Med. 44, 14-22 (2016).

9. Advani, S. D., Murray, T. S., Murdzek, C. M., Aniskiewicz, M. J. \& Bizzarro, M. J. Shifting focus toward healthcare-associated bloodstream infections: the need for neonatal intensive care unit-specific NHSN definitions. Infect. Control Hosp. Epidemiol. 41, 181-186 (2020).

10. Folgori, L., Bielicki, J. \& Sharland, M. A systematic review of strategies for reporting of neonatal hospital-acquired bloodstream infections. Archives of disease in childhood. Arch. Dis. Fetal Neonatal Ed. 98, F518-F523 (2013).

11. Lorch, S. A. National quality measures in perinatal medicine. Clin. Perinatol. 44, 485-509 (2017).

12. Goerens, A. et al. Neonatal ventilator associated pneumonia: a quality improvement initiative focusing on antimicrobial stewardship. Front. Pediatr. 6, 262-262 (2018).

13. Jacobs Pepin, B., Lesslie, D., Berg, W., Spaulding, A. B. \& Pokora, T. ZAP-VAP: a quality improvement initiative to decrease ventilator-associated pneumonia in the neonatal intensive care unit, 2012-2016. Adv. Neonatal Care 19, 253-261 (2019).

14. Azab, S. F. A. et al. Reducing ventilator-associated pneumonia in neonatal intensive care unit using "VAP prevention Bundle": a cohort study. BMC Infect. Dis. 15, 314-314 (2015).

15. Gokce, I. K. et al. Successful implementation of a bundle strategy to prevent ventilator-associated pneumonia in a neonatal intensive care unit. J. Trop. Pediatr. 64, 183-188 (2018).

16. Rogers, E., Alderdice, F., McCall, E., Jenkins, J. \& Craig, S. Reducing nosocomial infections in neonatal intensive care. J. Matern. Fetal Neonatal Med. 23, 1039-1046 (2010)

17. Weber, C. D. Applying adult ventilator-associated pneumonia bundle evidence to the ventilated neonate. Adv. Neonatal Care 16, 178-190 (2016).

18. Ceballos, K., Waterman, K., Hulett, T. \& Makic, M. B. F. Nurse-driven quality improvement interventions to reduce hospital-acquired infection in the NICU. Adv. Neonatal Care 13, 154-165 (2013).

19. Niedzwiecka, T. et al. What are the effects of care bundles on the incidence of ventilator-associated pneumonia in paediatric and neonatal intensive care units? A systematic review. J. Spec. Pediatr. Nurs. 24, e12264-e12264 (2019).

20. Hooven, T. A. \& Polin, R. A. Pneumonia. Semin. Fetal Neonatal Med. 22, 206-213 (2017). 